This is an electronic reprint of the original article. This reprint may differ from the original in pagination and typographic detail.

Author(s): Ilves, Outi; Häkkinen, Arja; Dekker, Joost; Pekkarinen, Liisa; Piitulainen, Kirsi; Järvenpää, Salme; Marttinen, Ilkka; Vihtonen, Kimmo; Neva, Marko H.

Title: Quality of life and disability: can they be improved by active postoperative rehabilitation after spinal fusion surgery in patients with spondylolisthesis? A randomised controlled trial with 12-month follow-up

Year: $\quad 2017$

Version:

Please cite the original version:

Ilves, O., Häkkinen, A., Dekker, J., Pekkarinen, L., Piitulainen, K., Järvenpää, S., Marttinen, I., Vihtonen, K., \& Neva, M. H. (2017). Quality of life and disability: can they be improved by active postoperative rehabilitation after spinal fusion surgery in patients with spondylolisthesis? A randomised controlled trial with 12-month followup. European Spine Journal, 26(3), 777-784. https://doi.org/10.1007/s00586-0164789-5

All material supplied via JYX is protected by copyright and other intellectual property rights, and duplication or sale of all or part of any of the repository collections is not permitted, except that material may be duplicated by you for your research use or educational purposes in electronic or print form. You must obtain permission for any other use. Electronic or print copies may not be offered, whether for sale or otherwise to anyone who is not an authorised user. 


\section{Quality of life and disability - can they be improved by active postoperative rehabilitation after spinal fusion surgery in patients with spondylolisthesis? A randomised controlled trial with 12-month follow-up.}

\section{Introduction}

Isthmic and degenerative spondylolisthesis are common spinal disorders leading to chronic back and leg pain. Lumbar spine fusion has been shown to be effective treatment in both of these disorders when compared to conservative treatment $[1,2]$. Although lumbar spine fusion surgery (LSF) reduce disability and improve healthrelated quality of life (HRQoL) in patients with several spinal disorders, they do not reach the level of normal population in disability of physical component score of HRQoL at the 1-year follow-up [3]. In these patients, the chronic low back pain may have affected the muscle functioning [4] and cardiorespiratory fitness [5]. In addition, fusion surgery itself leads to changes in spinal function [6-9].

Thus far, a limited number of randomised controlled trials (RCTs) have been published and the evidence of exercise therapy in restoring the spinal functioning and improving disability or HRQoL after fusion surgery is weak [10-16]. The commencement time and structure of the rehabilitation programs in previous RCT's varies $[10-14,17]$. Most trials were implemented close to time of surgery, starting preoperatively [13, 14], during hospital stay or soon after discharge $[10,12]$. The two trials, which have been measuring disability and health related quality of life, indicate that combination of exercises and cognitive-behavioral therapy is more effective than exercises alone, when implemented within first postoperative months [10, 12]. Because the existing evidence concerning exercise rehabilitation after LSF is limited and controversial, there is no consensus on the contents of effective rehabilitation program after LSF.

The purpose of the present randomized controlled trial was to study the effectiveness of the 12-month progressive exercise intervention compared to usual care on quality of life and disability during the intervention and at the 1-year follow-up in LSF patients with spondylolisthesis.

\section{Materials and methods}

This randomised controlled trial (RCT) was carried out in Tampere University Hospital and Central Finland Central Hospital, Finland. All patients aged over 18 years scheduled to have elective LSF surgery for isthmic or degenerative spondylolisthesis were eligible for the study. The exclusion criteria were severe cardiorespiratory or musculoskeletal disease, fracture, tumour, severe psychiatric disorder, extensive lower limb paresis, alcohol abuse and immediate complications after surgery (infection) that may prevent participation in postoperative rehabilitation. The sample size calculation was performed for the main outcome measure, pain (visual analogue scale), and it is explained in the study protocol [18]. Participant recruitment started in September 2009 and ended in September 2010 when a sufficient sample size had been achieved. This study was approved by the 
Ethics Committee of Tampere University Hospital and by the Ethics Committee of Central Finland Central Hospital.

Altogether, 104 consecutive adults were randomised into either the exercise group (EG) receiving active postoperative rehabilitation or to the usual care group (UCG) three months after the operation. Six participants dropped out before the start of the intervention (Fig. 1); thus, the number of participants was 98. Allocation to the EG or to the UCG was done randomly using computer-generated four-block randomisation lists, compiled by biostatistician. There were separate randomisation lists for isthmic and degenerative spondylolisthesis patients to ensure that both diagnoses were evenly represented between both intervention groups. Randomisation was concealed and was carried out in both hospitals by a study nurse who was not otherwise involved with the study. The data was collected by research assistants who were blinded to the treatment. The physiotherapists could not be blinded because of the nature of the study. To avoid confusion between the EG and UCG treatments, both study arms had their own physiotherapists in each hospital.

Surgical indications and techniques, and pre- and postoperative rehabilitation protocols, were standardized between both hospitals before the study. Six experienced consultant surgeons were involved to the trial and they performed part of the operations together, so the operation techniques were similar. All participants had posterolateral instrumented fusion which was performed using midline approach, and $21 \%$ also had posterior or transforaminal interbody fusion. All participants received similar pre- and postoperative instructions for up to three postoperative months during which they were encouraged to walk and perform light muscle exercises and stretching [18].

The 12-month progressive home-based exercise program of EG started three months postoperatively. The program consisted of back-specific and aerobic exercises, and fear avoidance-counselling given by physiotherapist. The exercises were taught to each participant individually according to the exercise protocol targeting for improving coordination, muscle strength and also for increasing physical activity (PA) by walking [18]. Participants performed all of the exercises independently at home and had booster sessions with the physiotherapist every second month (6 meetings in total). During booster sessions, possible barriers to physical activity were identified and discussed with the patient. Harmful, irrational beliefs and fears towards activity were corrected, and more support was given over the phone as needed. The patients' experiences from the previous phase of exercise program were reviewed, and the next phase of the back-specific program was determined, as well as new aerobic training (step amount) target levels. Patients were also given pictorial and written instructions for the exercises.

Patients in the UCG received one guidance session in physiotherapy three months after the surgery, consisting of instructions for home exercises. The instructed home exercise program included light muscle endurance exercises (abdominal muscles, back muscles and hip muscles) and stretching and balance training (one leg standing), with no progression. The participants were instructed to perform the home exercises three times a week. Participants were also given pictorial and written instructions for the exercises. 


\section{Outcome measures}

The socio-demographic and clinical data included age, weight, height, smoking status, length of education, employment status and duration of symptoms, and self-reported co-morbidities were evaluated preoperatively. Exercise compliance in the EG was assessed by exercise diaries. Exercise data were not collected from the UCG during the intervention period to avoid confusion between the two treatments. The outcomes were assessed at the baseline ( 3 months after surgery), at the end of the intervention (15 months after surgery) and one year after intervention (27 months after surgery).

Disability was measured by the Oswestry Disability Index (ODI), Finnish version 2.0 [19, 20]. The ODI contains 10 questions, and the score ranges from 0-100, with higher scores indicating more severe disability [20]. Health-related quality of life was assessed using the RAND-36-questionnaire. Eight dimensions were formulated from the RAND-36 questionnaire. The scaling of each dimension is from 0 to 100 . The higher the value is, the better the HRQoL [21, 22]. Age- and sex-adjusted RAND-36 reference values for the normal Finnish population were used as a reference [21]. Average low back and leg pain intensity for the previous seven days were assessed using a 100-millimetre visual analogue scale (VAS) [23].

\section{Statistics}

Statistical analysis was carried out by using IBM SPSS Statistics (version 20) and STATA 13 softwares. The results are expressed as mean with standard deviation (SD), median with interquartile range (IQR) or counts with percentages. The main outcomes are given with 95 percent confidence intervals (95\% CI). Comparisons between the groups in sociodemographic and clinical data were made by independent samples t-test, bootstrapped type t-test or Mann-Whitney U test for continuous variables; McNemar's test or Chi's square test were used in case of categorical distributions. The differences between the groups and changes over time in outcomes were investigated using mixed models with unstructured covariance structure and appropriate contrast. Data was analyzed with "intention to treat" (ITT) strategy. The Finnish reference values in RAND-36 dimensions were weighted to match the gender and age distribution of study population. The $95 \%$ CI for the RAND-36 dimensions were obtained by bias-corrected bootstrapping.

\section{Results}

No between-group differences in socio-demographic or clinical data occurred at the baseline, except control group had more musculoskeletal co-morbidities (Table 1). Most (74\%) of the participants were women, and the mean age was 59 years (range 32-84 years). According to the exercise diaries of the EG, the median [IQR] frequency of back-specific exercises was $2.5[1.9 ; 3.4]$ times per week during the first two months of the intervention, and $1.4[0.6 ; 1.9]$ times per week during the last two months $(\mathrm{p}<0.001)$.

There were no between-group differences in changes in any RAND-36 dimensions during the intervention or at the 1-year follow-up (Table 2). However, both groups improved significantly in the Physical Functioning and Role Physical dimensions during the intervention. Compared to the Finnish reference values for the Physical Functioning, Role Physical and Bodily Pain dimensions, the patients' scores were lower in both groups at 
baseline, and the EG also had lower values in the Social Functioning dimension. Both groups achieved the Finnish reference values in all dimensions by the 1-year follow-up (Fig. 2).

The ODI score decreased from 24 (12) to 18 (14) in the EG (p=0.003) and from 18 (12) to 13 (11) in the UCG $(\mathrm{p}=0.012)$ during the intervention (between groups $\mathrm{p}=0.69)$ (Table 2). At baseline, 54\% of the EG had an ODI score of 20 or higher. After the intervention 29\%, and at 1-year follow-up 25\% of the EG had an ODI score $\geq 20$. The respective proportions in the UCG were $36 \%, 25 \%$ and $28 \%$.

\section{Discussion}

The aim of this study was to investigate the effectiveness of the home-based progressive 12-month exercise program compared to usual care after lumbar spine fusion surgery in patients with spondylolisthesis. This study showed that disability and physical performance-related dimensions of the HRQoL improved in both groups. However, we did not detect any difference between the groups during the intervention or at the 1-year follow-up visit. At the 1-year follow-up visit, one-fourth of all participants still had at least moderate disability.

The exercise program of EG comprised of progressive neutral spine control exercises and aerobic training, but it also included individual discussions and counselling aiming to prevent irrational fears of movement or re-injury. This program was expected to reduce disability and to improve HRQoL in long-term follow-up. In this study, the EG followed a progressive exercise program with individual adjustments to determine the optimal level of intensity or to enable persons with different functional abilities to perform the program at home [18]. According to the HRQoL findings of the present study, the Role Physical and Physical Functioning dimensions improved significantly in both groups during the intervention, but the increase levelled out by the 1-year follow-up. The Role Physical dimension was very low at baseline in both groups, and it also had the highest increase during the intervention and throughout follow-up. In other words, usual care also efficiently enhanced these outcomes. The Vitality, Social Functioning, Role Emotional and Mental Health dimensions did not change during the intervention or at the post-intervention follow-up. The most importantly, participants in both groups reached the age- and sex-matched levels of the reference normal Finnish population two years postoperatively in the Physical Functioning, Role Physical, Bodily Pain and Social Functioning dimensions of the HRQoL.

In this study, all dimensions of the HRQoL, except the Role Physical dimension, were higher at baseline compared to the baseline in the study by Monticone et al. in 2014 [12]. In that study, there were significant differences between the two treatment groups in the changes in all HRQoL dimensions of the SF-36, meaning that the 4-week cognitive-behavioural therapy (CBT) with exercise training was more effective than the 4-week exercise training alone, when implemented within the first five postoperative weeks [12]. The later timing of the intervention in the present study may explain the smaller changes, as the surgery and early postoperative care already improved outcomes before the 12-month intervention started. Despite the shift in clinical practice towards earlier rehabilitation using fast-track strategies, Oestergaard et al. 2013 found that later timing (start 12 weeks postoperatively) of exercise rehabilitation has shown better results and cost-effectiveness than fast-track rehabilitation (start 6 weeks postoperatively) [24]. 
In the present study, the disability of UCG was lower than EG at the baseline, but the changes in disability were analogous between the groups during the intervention. Furthermore, at the 1-year follow-up, the achieved ODI levels were maintained, meaning that one-fourth of participants reported at least moderate disability. The future challenge will be to identify these patients in an earlier postoperative phase to offer them more individual rehabilitation interventions, while most of the LSF patients could possibly recover with subtler interventions. In previously published RCTs, the mean ODI scores showed severe disability (over 40) at baseline [10, 12], while in this study, the mean disability levels were moderate in the EG and minimal in the UCG at baseline. In the study by Abbott et al. [10], the baseline measurement was actually performed preoperatively when the patient's clinical status was worse than three months postoperatively, which was the time point used as a baseline of present study. In the study by Abbott et al., the ODI score had the most significant decrease during the first three postoperative months in both groups (psychomotor therapy and exercise therapy), but the difference between the groups remained unchanged after 2-3-years of follow-up, in favour of psychomotor therapy (mean difference of 9.8 points, $\mathrm{p}=0.011$ ) [10]. A similar trend is also seen in the study by Monticone et al. [12], suggesting that CBT together with exercise is a more effective treatment than exercise alone when investigating disability. In addition, Qualitative study about patient's postoperative experiences after interdisciplinary CBT as part of surgery rehabilitation suggests, that CBT as part of therapy may alter patient's pain perceptions and improve pain coping behaviour [25].

Only in the study of Christensen et al. the commencement time of intervention was same three months as in our study $[11,16]$. However, the length of intervention in that study was only eight weeks and exercise protocol of training group was more intensive than the protocol used in the present study. The intensity and length of the intervention is probably the explanation why the significant pain problem found in study of Christensen was not found in present study. Therefore, the long-term postoperative rehabilitation is not problematic according our study because of inferior results, instead it is controversial because of cost-effectiveness since the results were not superior when compared to the usual care group.

In the present study, we used patient-reported outcome measures (PROMs), which show the effect of the treatment for the patients. The novelty of this study is the long-term combined back-specific and healthenhancing home-exercise program, which is resource-friendly option. These can be considered as the strengths of this study, as well as the authentic clinical setting, which could be easily implemented in clinical practice. This study has some limitations as well. In both groups, the results of surgery were good and the patients had already started light exercises in the early recovery phase, before the actual exercise intervention started; thus, the outcomes had already improved by the starting time of the intervention. To avoid confusion between the treatments of the two groups, we did not monitor the activities of the UCG with exercise diaries during the intervention.

\section{Conclusion}


In conclusion, the disability and physical dimensions of the HRQoL improved in both groups during the intervention. The progressive 12-month home-exercise intervention, which commenced three months postoperatively, was not superior to usual care. However, one-fourth of participants had at least moderate disability at the 12-month follow-up, and therefore, postoperative rehabilitation may need to be composed of more individually supported and tailored interventions. 
1. Moller H, Hedlund R (2000) Surgery Versus Conservative Management in Adult Isthmic Spondylolisthesis: A Prospective Randomized Study: Part 1.25(13):1711-1715

2. Weinstein JN, Lurie JD, Tosteson TD, Hanscom B, Tosteson ANA, Blood EA, Birkmeyer NJO, Hilibrand AS, Herkowitz H, Cammisa FP, Albert TJ, Emery SE, Lenke LG, Abdu WA, Longley M, Errico TJ, Hu SS (2007) Surgical versus Nonsurgical Treatment for Lumbar Degenerative Spondylolisthesis. N Engl J Med 356(22):2257-2270

3. Pekkanen L, Neva MH, Kautiainen H, Dekker J, Piitulainen K, Wahlman M, Hakkinen A (2013) Disability and health-related quality of life in patients undergoing spinal fusion: a comparison with a general population sample. BMC Musculoskelet Disord 14:211-2474-14-211

4. van Dieën JH, Selen LPJ, Cholewicki J (2003) Trunk muscle activation in low-back pain patients, an analysis of the literature. 13(4):333-351

5. Smeets RJEM, Wittink H, Hidding A, Knottnerus JA (2006) Do Patients With Chronic Low Back Pain Have a Lower Level of Aerobic Fitness Than Healthy Controls?: Are Pain, Disability, Fear of Injury, Working Status, or Level of Leisure Time Activity Associated With the Difference in Aerobic Fitness Level? Spine 31(1):90-97

6. Motosuneya T, Asazuma TF, Tsuji TF, Watanabe HF, Nakayama YF, Nemoto K (2006) Postoperative change of the cross-sectional area of back musculature after 5 surgical procedures as assessed by magnetic resonance imaging.

7. Hyun SJ, Kim YB, Kim YS, Park SW, Nam TK, Hong HJ, Kwon JT (2007) Postoperative Changes in Paraspinal Muscle Volume: Comparison between Paramedian Interfascial and Midline Approaches for Lumbar Fusion. J Korean Med Sci 22(4):646-651

8. Fan S, Hu Z, Zhao F, Zhao X, Huang Y, Fang X (2010) Multifidus muscle changes and clinical effects of one-level posterior lumbar interbody fusion: minimally invasive procedure versus conventional open approach. 19(2):316-324

9. Tarnanen S, Neva MH, Hakkinen K, Kankaanpaa M, Ylinen J, Kraemer WJ, Newton RU, Hakkinen A (2014) Neutral Spine Control Exercises in Rehabilitation After Lumbar Spine Fusion. J Strength Cond Res 7:20182019-2025

10. Abbott AD, Tyni-Lenne R, Hedlund R (2010) Early rehabilitation targeting cognition, behavior, and motor function after lumbar fusion: a randomized controlled trial. Spine (Phila Pa 1976) 35(8):848-857

11. Christensen FB, Laurberg I, Bunger CE (2003) Importance of the back-cafe concept to rehabilitation after lumbar spinal fusion: a randomized clinical study with a 2-year follow-up. Spine (Phila Pa 1976) 28(23):25612569

12. Monticone M, Ferrante S, Teli M, Rocca B, Foti C, Lovi A, Brayda Bruno M (2014) Management of catastrophising and kinesiophobia improves rehabilitation after fusion for lumbar spondylolisthesis and stenosis. A randomised controlled trial. Eur Spine J 23(1):87-95

13. Nielsen PR, Andreasen J, Asmussen M, Tonnesen H (2008) Costs and quality of life for prehabilitation and early rehabilitation after surgery of the lumbar spine. BMC Health Serv Res 8:209-6963-8-209

14. Nielsen PR, Jørgensen LD, Dahl B, Pedersen T, Tønnesen H (2010) Prehabilitation and early rehabilitation after spinal surgery: randomized clinical trial. Clin Rehabil 24(2):137-148

15. Rushton A, Eveleigh G, Petherick EJ, Heneghan N, Bennett R, James G, Wright C (2012) Physiotherapy rehabilitation following lumbar spinal fusion: a systematic review and meta-analysis of randomised controlled trials. BMJ Open 2(4):10.1136/bmjopen-2012-000829. Print 2012 
16. Soegaard R, Christensen FB, Lauerberg I, Bunger CE (2006) Lumbar spinal fusion patients' demands to the primary health sector: evaluation of three rehabilitation protocols. A prospective randomized study. Eur Spine $\mathrm{J}$ 15(5):648-656

17. Soegaard R, Christensen FB, Christiansen T, Bunger C (2007) Costs and effects in lumbar spinal fusion. A follow-up study in 136 consecutive patients with chronic low back pain. Eur Spine J 16(5):657-668

18. Tarnanen S, Neva MH, Dekker J, Häkkinen K, Vihtonen K, Pekkanen L, Häkkinen A (2012) Randomized controlled trial of postoperative exercise rehabilitation program after lumbar spine fusion: study protocol. 13(1): 123

19. Pekkanen L, Kautiainen H, Ylinen J, Salo P, Hakkinen A (2011) Reliability and validity study of the Finnish version 2.0 of the oswestry disability index. Spine (Phila Pa 1976) 36(4):332-338

20. Fairbank J, Couper J, Davies J, O'Brien J (1980) The Oswestry low back pain disability questionnaire. $66: 271-273$

21. Aalto A, Aro AR, Teperi J (1999) RAND-36 terveyteen liittyvän elämänlaadun mittarina. Mittarin luotettavuus ja suomalaiset väestöarvot. STAKES Sosiaali- ja terveysalan tutkimus- ja kehittämiskeskus, Helsinki

22. Hays R, Sherbourne C, Mazel R (1993) The RAND 36-item health survey 1.0. (2):217-227

23. Price DD, McGrath PA, Rafii A, Buckingham B (1983) The validation of visual analogue scales as ratio scale measures for chronic and experimental pain. Pain 17(1):45-56

24. Oestergaard L, Christensen F, Nielsen C, Bunger C, Fruensgaard S, Sogaard R (2013) Early versus late initiation of rehabilitation after lumbar spinal fusion: economic evaluation alongside a randomized controlled trial. 38(23):1979-1985

25. Lindgreen P, Rolving N, Nielsen CV, Lomborg K (2016) Interdisciplinary Cognitive-Behavioral Therapy as Part of Lumbar Spinal Fusion Surgery Rehabilitation: Experience of Patients With Chronic Low Back Pain. 35(4):238-247 
Table 1. Baseline characteristics of the participants.

\begin{tabular}{|c|c|c|c|}
\hline & $\begin{array}{c}\text { EG } \\
\mathrm{N}=48\end{array}$ & $\begin{array}{l}\text { UCG } \\
\mathbf{N}=\mathbf{5 0}\end{array}$ & $\begin{array}{c}\text { p-value between } \\
\text { groups }\end{array}$ \\
\hline Women, n (\%) & $34(71)$ & $38(76)$ & 0.56 \\
\hline Age, years, mean (SD) & $59(12)$ & $58(12)$ & 0.59 \\
\hline Body mass index, mean (SD) & $28.3(4,8)$ & $28.3(4.8)$ & 0.99 \\
\hline Smokers, n (\%) & $9(19)$ & $6(12)$ & 0.58 \\
\hline Length of education, years, mean (SD) & $12.0(3.7)$ & $12.6(3.6)$ & 0.41 \\
\hline Work status, $\mathrm{n}(\%)$ : & & & 0.27 \\
\hline Working & $17(35)$ & $12(24)$ & \\
\hline Temporarily not working & $10(21)$ & $17(34)$ & \\
\hline Retired & $21(44)$ & $21(42)$ & \\
\hline Primary diagnosis n (\%): & & & 0.72 \\
\hline Degenerative spondylolisthesis & $32(67)$ & $35(70)$ & \\
\hline Isthmic spondylolisthesis & $16(33)$ & $15(30)$ & \\
\hline Duration of current symptoms before surgery, months, mean (SD) & $41(37)$ & $40(36)$ & 0.80 \\
\hline \multicolumn{4}{|l|}{ Self-reported comorbidities, n (\%): } \\
\hline Blood pressure & $24(51)$ & $25(51)$ & 0.99 \\
\hline Diabetes & $3(6)$ & $6(12)$ & 0.49 \\
\hline Other musculoskeletal disorders & $4(9)$ & $13(27)$ & 0.03 \\
\hline Neurological disorders & $2(4)$ & $1(2)$ & 0.61 \\
\hline Mental health disorders & $2(4)$ & $1(2)$ & 0.61 \\
\hline Pulmonary disorders & $4(9)$ & $6(12)$ & 0.74 \\
\hline Cardiovascular disorders & $3(6)$ & $5(10)$ & 0.71 \\
\hline
\end{tabular}

$\mathrm{EG}=$ exercise group, $\mathrm{UCG}=$ usual care group, $\mathrm{SD}=$ standard deviation, $\mathrm{IQR}=$ inter-quartile range 

Table 2. Baseline scores and changes in the RAND-36 dimensions during the intervention and at follow-up, reported as the means with standard deviation (SD) or 95 percent confidence intervals $(95 \% \mathrm{CI})$. The changes are based on mixed model estimates. Between group p-values are adjusted by age and sex.

\begin{tabular}{|c|c|c|c|c|c|c|c|c|}
\hline & \multicolumn{2}{|c|}{ Baseline } & \multicolumn{2}{|c|}{ Change during intervention } & \multirow{2}{*}{$\begin{array}{c}\mathrm{p} \text {-value between } \\
\text { the groups }\end{array}$} & \multicolumn{2}{|c|}{ Change during 1-year follow-up } & \multirow{2}{*}{$\begin{array}{l}\mathrm{p} \text {-value betweer } \\
\text { the groups }\end{array}$} \\
\hline & EG & UCG & EG & UCG & & EG & UCG & \\
\hline & Mean (SD) & Mean (SD) & Mean $(95 \% \mathrm{CI})$ & Mean $(95 \% \mathrm{CI})$ & & Mean $(95 \%$ CI) & Mean $(95 \% \mathrm{CI})$ & \\
\hline \multicolumn{9}{|l|}{ RAND-36: } \\
\hline Physical functioning & $59.6(20.4)$ & $67.1(17.4)$ & $10.0(4.6$ to 15.3$)$ & $7.8(2.5$ to 13.0$)$ & 0.53 & $-0.5(-4.8$ to 3.7$)$ & $-1.4(-5.5$ to 2.6$)$ & 0.75 \\
\hline Role physical & $22.3(31.7)$ & $38.5(36.8)$ & $20.0(7.7$ to 32.3$)$ & 16.4 (4.4 to 28.4$)$ & 0.67 & $9.9(-3.6$ to 23.3$)$ & $5.7(-7,2$ to 18.5$)$ & 0.65 \\
\hline Bodily pain & $55.1(20.7)$ & $60.8(19.3)$ & $5.3(-1.7$ to 12.4$)$ & $6.3(-0.6$ to 13.1$)$ & 0.88 & $1.5(-5.4$ to 8.3$)$ & $1.5(-5.0$ to 8.0$)$ & 0.99 \\
\hline General health & $62.4(20.4)$ & $59.4(19.0)$ & $-2.6(-7.1$ to 2.0$)$ & $1.3(-3.2$ to 5.7$)$ & 0.23 & $2.7(-2.2$ to 7.6$)$ & $-0.8(-5.4$ to 3.8$)$ & 0.30 \\
\hline Vitality & $61.1(24.0)$ & $65.8(18.5)$ & $1.3(-4.7$ to 7.4$)$ & $-1.9(-7.8$ to 4.1$)$ & 0.44 & $-1.1(-6.9$ to 4.6$)$ & $0.1(-5.4$ to 5.7$)$ & 0.74 \\
\hline Social functioning & $71.5(25.0)$ & $79.7(20.2)$ & $3.0(-4.9$ to 10.9$)$ & $5.4(-2.3$ to 13.1$)$ & 0.68 & $7.9(1.0$ to 14.8$)$ & $-1.2(-7.7$ to 5.4$)$ & 0.062 \\
\hline Role emotional & $60.1(44.2)$ & $68.1(40.1)$ & $2.1(-16.6$ to 20.8$)$ & $4.9(-8.5$ to 18.3$)$ & 0.86 & $1.2(-11.1$ to 13.5$)$ & $-5.6(-17.2$ to 6.0$)$ & 0.43 \\
\hline Mental health & $74.0(19.5)$ & $77.1(16.6)$ & $-0.4(-5.4$ to 4.7$)$ & $-1.2(-6.2$ to 3.7$)$ & 0.81 & $2.1(-2.7$ to 6.9$)$ & $-1.0(-5.6$ to 3.6$)$ & 0.36 \\
\hline ODI & $24.0(12.0)$ & $17.8(11.7)$ & $-5.6(-9.2$ to -1.9$)$ & $-4.6(-8.2$ to -1.0$)$ & 0.69 & $-1.2(-3.9$ to 1.3$)$ & $2.1(-0.4$ to 4.5$)$ & 0.065 \\
\hline Back pain (VAS) & $21.1(17.9)$ & $17.3(18.0)$ & $-1.6(-6.6$ to 3.7$)$ & $3.8(-0.9$ to 8.8$)$ & 0.16 & $2.0(-3.2$ to 7.5$)$ & $4.4(-2.5$ to 10.7$)$ & 0.64 \\
\hline Leg pain (VAS) & $18.8(22.2)$ & $14.3(20.0)$ & $4.1(-4.4$ to 11.7$)$ & $0.4(-6.4$ to 5.8$)$ & 0.38 & $3.3(-1.1$ to 8.4$)$ & $5.1(-3.2$ to 13.2$)$ & 0.71 \\
\hline
\end{tabular}

$\mathrm{EG}=$ exercise group, $\mathrm{UCG}=$ usual care group, $95 \% \mathrm{CI}=95 \%$ confidence interval, $\mathrm{ODI}=$ Oswestry Disability Index, VAS=Visual analogue scale 



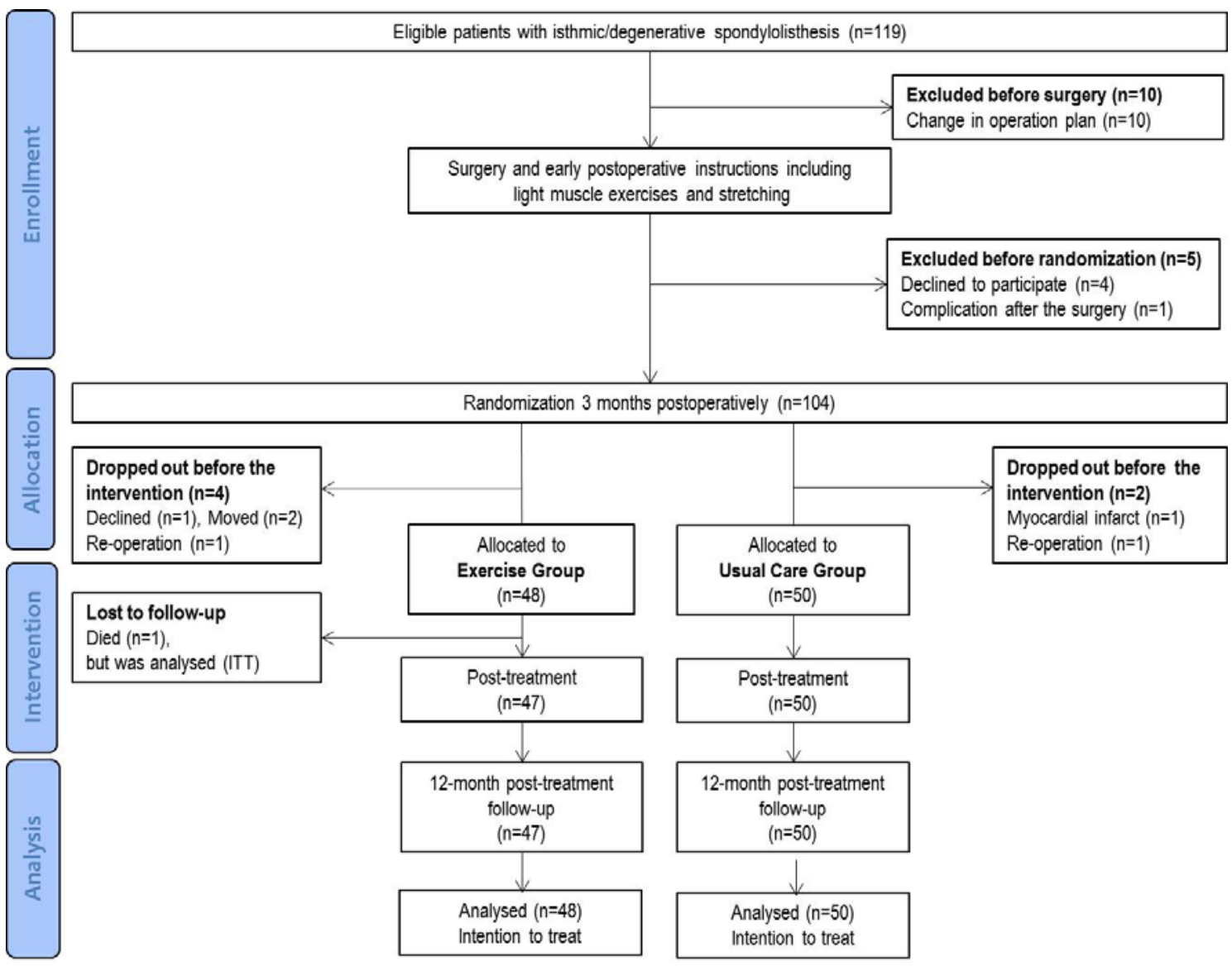

Figure 1. Participant flow diagram 

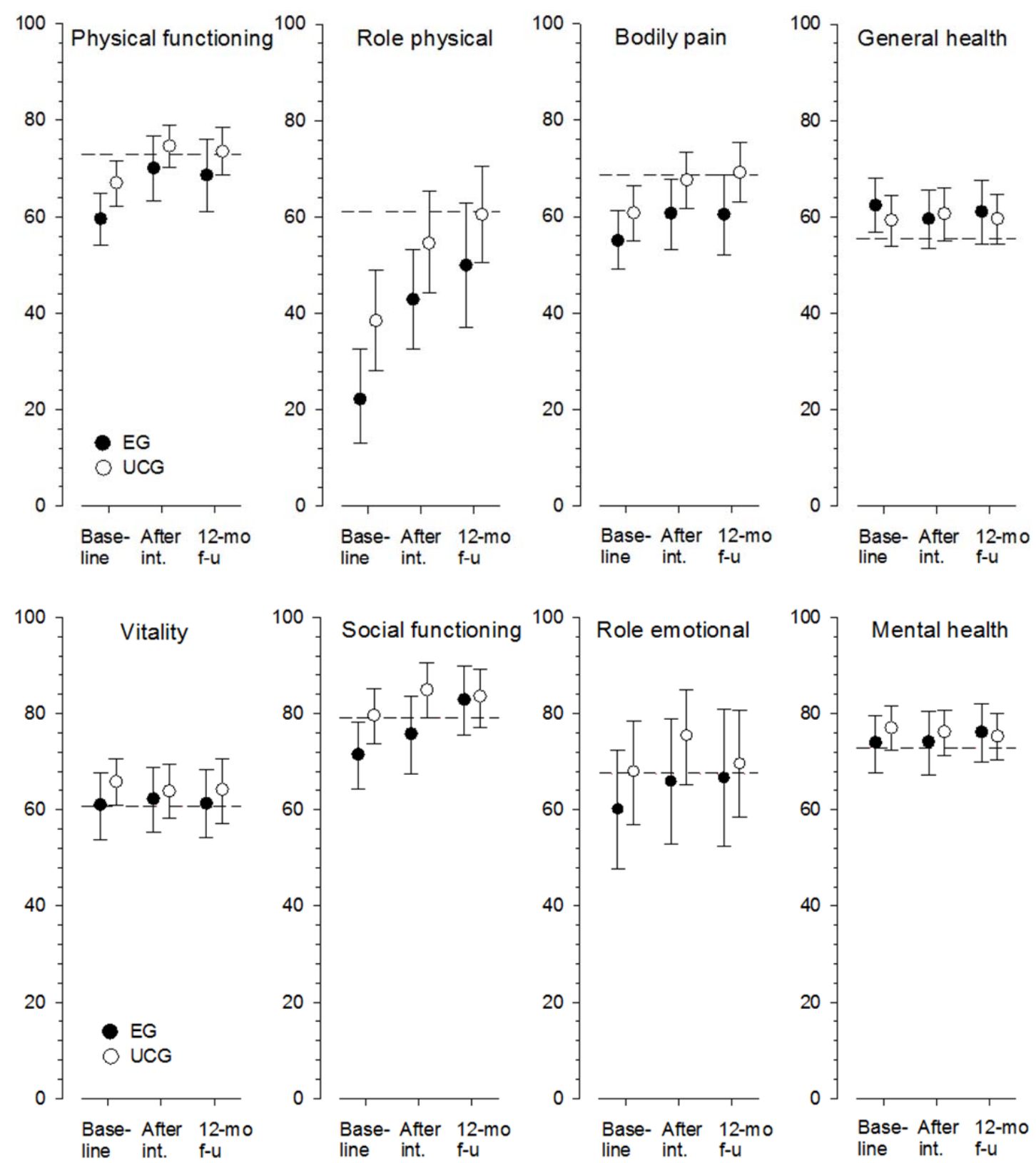

Figure 2. The-RAND-36 dimensions (mean with 95\%CI) by group at each measurement point. The dashed lines show the Finnish population reference values. $\mathrm{EG}=$ exercise group, $\mathrm{UCG}=$ usual care group, After int. $=$ after intervention, 12-mo $\mathrm{f}$-u=12-month follow-up 